\title{
Índices de Cysticercus tenuicollis (Taenia hydatigena cysts) en cavidad abdominal de ovinos faenados en
}

\author{
el camales frigoríficos municipales
}

Indices of Cysticercus tenuicollis (Taenia hydatigena cysts) in abdominal cavity of sheep slaughtered in municipal slaughterhouses

\author{
Almeida-Secaira Roberto Ismael ${ }^{*}$, Núñez-Torres Oscar Patricio, Cuadrado-Guevara Camila Andrea, \\ Vargas-Secaira Christian Javier
}

\begin{tabular}{l}
\hline Datos del Artículo \\
\hline Universidad Técnica de Ambato. \\
Facultad de Ciencias Agropecuarias. \\
Cantón Cevallos. \\
Tungurahua - Ecuador. \\
Casilla postall 18-01-334. \\
Telf: (+593)032872630-0985471191 \\
*Dirección de contacto: \\
Universidad Técnica de Ambato. \\
Facultad de Ciencias Agropecuarias. \\
Cantón Cevallos. Tungurahua. \\
Ecuador. Casilla postal: 18-01-334. \\
Telf: (+593) 032746151 - 032746171 \\
Roberto Ismael Almeida Secaria \\
E-mail address : ri.almeida@uta.edu.ec \\
\hline \\
Palabras clave: \\
Cavidad abdominal, \\
cisticerco, \\
epiplón, \\
frecuencia, \\
parásito
\end{tabular}

\section{J. Selva Andina Anim Sci.} 2017; 4(2):104-115.

\section{Historial del artículo. \\ Recibido abril, 2017. \\ Devuelto junio 2017 \\ Aceptado septiembre, 2017 \\ Disponible en línea, octubre, 2017.}

\begin{tabular}{c}
\hline Editado por: \\
Selva Andina \\
Research Society \\
\hline
\end{tabular}

\section{Resumen}

El propósito de Esta investigación fue el de determinar la prevalencia de Cysticercus tenuicollis e identificar las variables que pueden influir para que la enfermedad parasitaria se presente en el ganado ovino. En el estudio se cuantifico una población total de 362 animales los cuales fueron examinados durante el proceso de faenamiento, un total de 133 ovinos fueron positivos a la presencia del cisticerco del modo que se obtuvo una frecuencia de periodo del 37\% en la población. En la investigación se determinó que no existe dependencia de la raza, sexo y procedencia de los animales para la presentación del parásito en su interior. Se identificó la distribución del parásito en la cavidad abdominal de los ovinos faenados determinando que el epiplón es la estructura anatómica mayormente afectada por la presencia del C. tenuicollis, entre los órganos afectados se encuentran: hígado, mesenterio y peritoneo con un diámetro promedio por cisticerco de entre 2.5 a $3.7 \mathrm{~cm}$ dependiendo del órgano o estructura infestada. Mediante el análisis estadístico $\mathrm{Chi}^{2}$ se determinó que el parásito C. tenuicollis afecta a todos y cada uno de los animales sin tener diferencia de razas, sexo y procedencia puesto que la probabilidad de ocurrencia en las tres variables propuestas es superior a la establecida y el parásito no difiere con ninguna de las categorías antes descritas.

() 2017. Journal of the Selva Andina Animal Science. Bolivia. Todos los derechos reservados.

\section{Abstract}

The purpose of this research was to determine the prevalence of Cysticercus tenuicollis and to identify the variables that may influence parasitic disease in sheep. The study quantified a total population of 362 animals which were examined during the slaughter process, a total of 133 sheep were positive to the presence of the cysticercus so that a period frequency of $37 \%$ was obtained in the population. In the investigation it was determined that there is no dependence of the race, sex and origin of the animals for the presentation of the parasite inside. The distribution of the parasite was identified in the abdominal cavity of sheep slaughtered, determining that the omentum is the anatomical structure most affected by the presence of $C$. tenuicollis, among the affected organs are: liver, mesentery and peritoneum with an average diameter by cysticercus between 2.5 and $3.7 \mathrm{~cm}$ depending on the organ or structure infested. Statistical analysis Chi2 determined that the parasite $C$. tenuicollis affects each and every one of the animals without differences of races, sex and origin since the probability of occurrence in the three variables proposed is higher than that established and the parasite does not differs with any of the categories described above.

Key words:

Abdominal cavity,

cysticercus,

omentum,

frequency, 


\section{Introducción}

Debido a que en el país, en especial las zonas en donde se cría el ganado ovino ya sea para carne, lana $\mathrm{u}$ otros productos y subproductos, existe un desconocimiento acerca de la enfermedad parasitaria que produce el Cysticercus tenuicollis al ganado ovino, por ende dicha población se encuentra propensa a adquirir el parásito. Ciertas personas conocen el parásito porque han tenido la oportunidad de verlo en camales o cuando los ovinos son sacrificados cerca de su granja o dentro de la misma ya que el ganado ovino es de fácil sacrificio y es la principal fuente de alimento en las familias, las personas que han visto el $C$. tenuicollis en la canal del animal los denominan "vegijas de agua". La influencia del hombre ha permitido que este animal ha ido evolucionando además de la selección natural que se da en el planeta y sus condiciones geográficas, estos mamíferos detallan de importancia para los humanos ya que se adaptan bien a climas tanto tropicales como fríos con diferentes texturas de terreno. Se puede obtener mucho provecho ya que este animal puede ser utilizado para obtener productos y subproductos entre ellos está: la venta de animales, carne para consumo familiar e industrial, cueros para prendas de vestir entre otros. (Atto-Mendives 2007).

El ganado ovino es un tipo de animal muy rustico a la vez que se adapta muy bien en la mayoría de terrenos, capaz de consumir cualquier planta y sacarle provecho nutricional por su excelente conversión alimenticia que posee pero, los costes de producción debido a las enfermedades en una explotación pueden llegar a ser lo suficientemente importantes como para hacerla inviable económicamente, entre las enfermedades de importancia para la producción se encuentran las enfermedades parasita 105 rias, como: hidatidosis y cisticercosis ya que muchas veces pasan desapercibida por la baja mortalidad que suelen conllevar, pero cuya influencia en la producción está ampliamente demostrada y constatada. (Frontera-Carrión et al. 2012).

Realizando una comparación con otro mamífero se tiene claro que el ganado ovino posee una serie de ventajas importantes sobre los bovinos que son los mamíferos mayormente explotados por la demanda de productos a nivel mundial que existe, una de estas ventajas es que: poseen una mayor capacidad reproductiva (dos partos por año), con un intervalo entre partos entre los 6 y 7 meses, a diferencia de los bovinos estos por lo general tienen mayor número de crías por parto, siendo normal gemelos o trillizos, el ganado ovino posee la mayor capacidad de conversión alimenticia, es decir, comer menos en volumen y producir más en carne, lana o leche, al ser un animal rustico las ovejas tienden a ser más resistentes a las alturas, menor precio de compra por animal, tienen la posibilidad de usarse para el consumo de la familia por su fácil sacrificio, mayor calidad en la carne, que dependerá igualmente del proceso de crianza y manejo del animal, mayor calidad en la leche para derivados como el queso, mayor calidad en la piel para procesos de curtiembre. (Vega-Pérez \& Garcia-Barrera 2011).

El ganado ovino puede consumir mucho mejor los pastos y forrajes, ya que pueden utilizar 1.5 a 2 veces más las plantas que el ganado mayor y son capaces de consumir 540 especies de hierbas entre las 600 existentes, esto se debe a la gran movilidad y a las características de su tracto digestivo, pueden pastar en áreas ya utilizadas por otros animales domésticos, siendo los pastos inmaduros los más nutritivos para los animales, bien pastados en el campo, 
ensilados o desecados. De acuerdo a esto lo que lo vuelve más rentable que otro tipo de ganado (VegaPérez \& Garcia-Barrera 2011).

Las enfermedades parasitarias por lo general provocan de una u otra forma que al animal afectado, vaya disminuyendo paulatinamente su capacidad de producción, la cual es apreciable de acuerdo a la sintomatología que se presenta. Una enfermedad aguda puede producir alta y rápida mortalidad, en cambio en un cuadro crónico se observa una disminución de la producción por lo general acompañada de signos clínicos como anorexia, diarrea y enflaquecimiento (González 1982). Con el nombre genérico de cisticercosis de los animales domésticos se conoce a infestaciones causadas por la presencia y acción de varias especies de estados larvarios de Taenia sp. En diferentes tejidos de bovinos, ovinos, caprinos, porcinos, equinos, etc. La fuente de infestación la constituyen principalmente el hombre, perros y gatos que actúan como huéspedes definitivos. (Quiroz-Romero 1984). El cestodo Taenia hydatigena se encuentra en el intestino delgado de perros, lobos, coyotes, zorros y otros cánidos, sin embargo, la fase larvaria, conocida como $C$. tenuicollis se encuentra en el hígado, mesenterio, de bovinos ovinos caprinos y otros animales como el cerdo. (Quiroz-Romero et al. 2011). El parásito adulto que es la $T$. hydatigena, puede llegar a medir de 75 hasta los $500 \mathrm{~cm}$ de largo el cual se aloja a lo largo del intestino delgado del perro u otros carnívoros. En su parte inicial consta de un róstelo el cual tiene de 26 a 44 ganchos en una corona doble lo cual le permite al parásito adherirse a la mucosa del intestino en donde absorbe los nutrientes necesarios para su desarrollo, los ganchos grandes miden de 0.17 a $0.22 \mathrm{~mm}$ de largo, los pequeños de 0.11 a $0.16 \mathrm{~mm}$. Los proglótidos grávidos miden de $10 \mathrm{a}$ $14 \mathrm{~mm}$ de largo por 4 y $7 \mathrm{~mm}$ de ancho, y el útero presenta entre 5 a 10 ramas sobre uno y otro lado.
Los huevos son elípticos y miden entre 38 a 39 por 34 a 35 micras. (Quiroz-Romero 1984). El objetivo de la investigación fue determinar mediante la inspección post-mortem la prevalencia de $C$. tenuicollis en cavidad abdominal en ovinos faenados en el camal municipal de Ambato.

\section{Materiales y métodos}

Esta investigación se realizó con un enfoque cualitativo en donde se observó la presencia del C. tenuicollis en los órganos y estructuras afectadas y a su vez un enfoque cuantitativo debido a los resultados obtenidos los cuales se expresaran en los análisis de frecuencias y porcentuales. Utilizando una modalidad mixta (de campo, de laboratorio). Para esta investigación se utilizó una modalidad mixta, de campo, debido a que se necesitó encuestar a los comerciantes o propietarios sobre la raza y el origen de los ovino previo a su faenamiento en los corrales del establecimiento, en el momento de faenamiento se observaba minuciosamente los órgano y vísceras del ovino para determinar la presencia de $C$. tenuicollis, y también una modalidad de laboratorio, debido a que después de presenciar al parásito se lo recolectaba las vesículas presentes en una bolsa hermética para su verificación en el laboratorio de médicos veterinarios del camal, para su respectiva verificación y para la toma de diámetro de los C. tenuicollis, se tomó al azar una vesícula con el escólex desarrollado para observarlo determinadamente bajo el microscopio utilizando violeta de genciana. Esta investigación es del tipo exploratorio ya que para determinar la raza y la procedencia se acudía a las instalaciones en donde se receptan el ganado ovino y es una investigación descriptiva ya que en cada uno de los datos se da una característica individual. 
El Camal Frigorífico Municipal de la ciudad de Ambato, Provincia del Tungurahua, región sierra, localizado en el parque industrial en la Parroquia Izamba sector el Pisque a $5 \mathrm{~km}$ de la mencionada localidad por la panamericana Norte, limita con las Parroquias de Unamuncho, río Ambato, Atahualpa y el río Culapachán. (Borja-Caicedo 2013) El factor en estudio fue el $C$. tenuicollis en animales infectados según sea su: raza, sexo y procedencia.

El universo de esta investigación consta de absolutamente todos los ovinos faenados en el Camal Frigorífico Municipal de Ambato, al cual ingresaron diferentes, razas, sexo, y provenientes de las principales ferias y sectores en donde se cría y produce este tipo de ganado.

De acuerdo con la investigación realizada se tomó en cuenta a todos y cada uno de los individuos en cuestión para poder recoger datos pertinentes a la investigación, la población estuvo constituida por ovinos faenados en el Camal Frigorífico Municipal de Ambato que acuden diariamente a este centro de faenamiento en horarios preestablecidos por la administración y personal adjunto. Para la investigación realizada se utilizó todos los elementos que ingresaron a el faenamiento los días respectivos debido a que mientras mayor sean los animales faenados mayor será el rango de eficiencia de la investigación la cual se realizó en el periodo de 23 de Febrero a 31 Mayo del 2015 para determinar la incidencia del parasito, siendo 362 el total de animales sacrificados.

En el trabajo de campo realizado se observó que razas son las que llegaron con mayor frecuencia al camal frigorífico municipal del cantón Ambato para su sacrificio, en una inspección ante-morten en los corrales, fuera de las instalaciones de faenamiento, se verifico las razas, los cuales se identificaron gracias a sus características externas que cada indivi107 duo presentaba, también se pudo constatar en el momento del faenamiento el sexo de los animales sacrificados para determinar si el género interviene en la infección del parasito, además mediante las guías de movilización de ganado, encuestas personales a productores y comerciantes se pudo obtener información de donde provenían los ovinos que fueron faenados en diferentes días.

En el trabajo realizado, se pudo observar mediante la inspección macroscópica de los órganos internos durante el proceso de evisceración del animal, en este punto la revisión se determina que órganos son aptos para el consumo humano, por ello en la investigación se verifico la presencia de vesículas de $C$. tenuicollis en órganos y estructuras dentro de la cavidad abdominal del animal, en donde se determinaba si el parasito provocaba o no alguna lesión en dicho órgano que contenía la vesícula o por donde el parásito había circulado.

Durante el proceso de evisceración de la parte abdominal de ganado ovino sacrificado, en cada individuo se recogía las vesículas presentes y de manera aislada se los ubicaba para su correcta medición del diámetro del C. tenuicollis con la ayuda de un calibrador utilizado para la medición de diámetros.

Después de terminado el trabajo de campo, la información que fue recolectada en hojas diseñadas por el autor, para recopilar los datos necesarios para un correcto desarrollo del proyecto, en el cual antes, durante y después del faenamiento se fueron recolectando información necesaria para poder cumplir los objetivos establecidos. Posteriormente pasa a verificar y tabular la información obtenida para un correcto análisis demostrado en: frecuencias absolutas y porcentuales con sus respectivos gráficos además de los análisis estadísticos para determinar la hipótesis y la asociación o independencia de las variables. 
Para determinar la prevalencia de la investigación se tomó en cuenta el método estadístico utilizado en epidemiologia que es: la prevalencia de periodo, se define como la frecuencia de una enfermedad o condición existentes, durante un lapso definido, tal como un año. También se refiere a una proporción que expresa la probabilidad de que un individuo sea un caso en cualquier momento de un determinado periodo de tiempo. (Kahl-Martin 1990).

La prevalencia de periodo se estima con la siguiente fórmula:

\section{Prevalencia de periodo $P P($ to, $t)=C($ to, $t) / N$}

$\mathrm{C}(\mathrm{to}, \mathrm{t})=$ número de casos incidentes o prevalentes identificados durante el periodo de tiempo. $\mathrm{N}=$ es el tamaño de la población. Su valor dependerá del tipo de población observada: población transversal, población estable o cohorte fija.

Para la verificación de la hipótesis planteada y determinar si existe significancia para la asociación o independencia entre la frecuencia de casos y las variables raza, sexo y procedencia, se lo determino usando el método estadístico de Chi 2 con un intervalo de confianza al $95 \%$ con una significancia del 0,05. Para lo cual se llevó a cabo la cuantificación de casos positivos, además se utilizó el cálculo de frecuencias absolutas y porcentuales para ser representados en histogramas de barras.
De igual manera se utilizó el Chi 2 para determinar la asociación o independencia entre la variable raza y sexo en conjunto con los órganos o estructuras más afectadas que se encuentren en la investigación. Además se utilizó el cálculo de frecuencias absolutas y porcentuales para ser representado en diagramas de sectores.

$$
X^{2}=\Sigma \frac{(O-E)^{2}}{E}
$$

$\mathrm{X}^{2}=$ Test estadístico Chi cuadrado. = Sumatoria de $\mathrm{O}=$ Frecuencias Observadas $\mathrm{E}=$ Frecuencias Esperadas.

Los materiales utilizados Biológicos fueron ovinos faenados y vesículas de $C$. tenuicollis y físicos como materiales de diagnóstico: (guantes de diagnóstico, tijeras, bisturí, pinzas, cuchillos), materiales de seguridad: equipo de protección (overol, mandil, casco, botas, visores, cofia, mascarilla, protectores auditivos, gel antiséptico) y materiales de escritorio: (regla, calibrador, hojas de campo, esferográficos, lápiz, borrador, carpeta, tableros, calculadora, computadora, cámara digital.

\section{Resultados}

Tabla 1 Cuantificación y análisis porcentuales de casos positivos en ovinos faenados, de acuerdo a la raza

\begin{tabular}{lcccccc}
\hline \multirow{2}{*}{ Raza } & \multicolumn{7}{c}{ Ovinos } \\
\cline { 2 - 7 } & \multicolumn{2}{c}{ positivos } & \multicolumn{2}{c}{ negativos } & Total & Total \\
\cline { 2 - 7 } & Frecuencia & \% & Frecuencia & $\%$ & Frecuencia & \% \\
\cline { 2 - 6 } Criolla & 65 & 38.69 & 103 & 61.31 & 168 & 46.41 \\
Merino & 35 & 32.11 & 74 & 67.89 & 109 & 30.11 \\
Mestiza & 21 & 41.18 & 30 & 58.82 & 51 & 14.09 \\
Corriedale & 12 & 35.29 & 22 & 64.71 & 34 & 9.39 \\
\hline \multicolumn{1}{r}{ Total } & 133 & & 229 & & 362 & 100.00 \\
\hline
\end{tabular}


Tabla 2 Cuantificación y análisis porcentual de casos positivos en ovinos faenados, de acuerdo al sexo

\begin{tabular}{|c|c|c|c|c|c|c|}
\hline \multirow{3}{*}{ Sexo } & \multicolumn{6}{|c|}{ Ovinos } \\
\hline & \multicolumn{2}{|c|}{ positivos } & \multicolumn{2}{|c|}{ negativos } & \multirow{2}{*}{$\begin{array}{c}\text { Total } \\
\text { Frecuencia }\end{array}$} & \multirow{2}{*}{$\begin{array}{c}\text { Total } \\
\%\end{array}$} \\
\hline & Frecuencia & $\%$ & Frecuencia & $\%$ & & \\
\hline Macho & 56 & 42.42 & 76 & 57.58 & 132 & 36.46 \\
\hline Hembra & 77 & 33.48 & 153 & 66.52 & 230 & 63.54 \\
\hline Total & 133 & & 229 & & 362 & 100.00 \\
\hline
\end{tabular}

Tabla 3 Cuantificación y análisis porcentuales de ovinos faenados. De acuerdo a la procedencia

\begin{tabular}{lcccccc}
\hline \multirow{2}{*}{ Procedencia } & \multicolumn{9}{c}{ Ovinos } \\
\cline { 2 - 7 } & \multicolumn{2}{c}{ positivos } & \multicolumn{2}{c}{ negativos } & Total & Total \\
\cline { 2 - 7 } & Frecuencia & \% & Frecuencia & \% & Frecuencia & \% \\
\cline { 2 - 7 } Izamba & 37 & 35.58 & 67 & 64.42 & 104 & 29.73 \\
Guaranda & 11 & 36.67 & 19 & 63.33 & 30 & 8.29 \\
Pelileo & 10 & 40.00 & 15 & 60.00 & 25 & 6.91 \\
Pilahuin & 8 & 34.78 & 15 & 65.22 & 23 & 6.35 \\
Quisapincha & 39 & 41.94 & 54 & 58.06 & 93 & 25.69 \\
Salcedo & 24 & 35.82 & 43 & 64.18 & 67 & 18.51 \\
Santa Rosa & 4 & 20.00 & 16 & 80.00 & 20 & 5.52 \\
\hline \multicolumn{1}{c}{ Total } & 133 & \multicolumn{7}{c}{} & 362 & 100.00 \\
\hline
\end{tabular}

Como se puede observar en el tabla 1. De un total de 362 ovinos faenados se determinó: 168 animales pertenecen a la raza Criolla, 65 positivos $38.69 \%$, mientras que, 109 animales pertenecen a la raza Merino 35 positivos $32.11 \%$, 51 animales pertenecen a la raza Mestiza 21 son positivos $41.18 \%, 34$ animales pertenecen a la raza Corriedale 12 positivos $35.29 \%$, razas que son faenadas en el camal municipal de Ambato. Del total de animales faenados tabla 2, de acuerdo al sexo de los animales, las hembras presentan un total de 77 casos positivos $33.48 \%$, de un total de 230 hembras ovinas, en tanto los machos presentaron 56 casos positivos de 132 animales $42.42 \%$ de machos que faenados en una población total de 362 animales.

En la tabla 3 las frecuencias de los 133 casos positivos en general distribuidos en lugares determinados: Izamba con 37 positivos $35.58 \%$, Guaranda con 11 casos $36.67 \%$, Pelileo 10 positivos $40.00 \%$, Pilahuin 8 positivos $34.78 \%$, el $41.94 \%$ posee Quisapincha con 39 animales positivos, San Miguel de Salcedo 109
$35.82 \%$ con 24 animales afectados, y por último Santa Rosa con 4 animales afectados $20.00 \%$. Determinando que estos lugares son la procedencia del ganado ovino.

Mediante la prueba estadística (Chi cuadrado), se determinó que el valor de $\mathrm{X}^{2}$ calculado es de 6.79, valor que es menor al de $\mathrm{X}^{2}$ tabulado que es de 16.92 con 9 GL. Por consiguiente se determina que la raza de los ovinos faenados no influye en la prevalencia de $C$. tenuicollis, afectando los mismos órganos o estructuras independientemente.

Mediante la cuantificación de los datos recolectados en el tabla 4 nos indica que en cada una de las razas estudiadas existe la presencia del $C$. tenuicollis determinando así que la raza criolla es afectada mayormente en el epiplón puesto que se detectó el parásito en 52 ovinos, en hígado 3 infestados, mesenterio 14 peritoneo 13. La raza Merino también presenta la mayor frecuencia en epiplón 29, hígado 4, 8 en mesenterio y 7 en peritoneo. La raza Mestiza presento el parásito con mayor frecuencia en epi- 
plón 17 infestados, en hígado no hallo, en mesenterio 5 individuos y 2 en peritoneo. Por último la raza Corriedale presenta más afectados en epiplón con 11 animales, 1 ovino positivo en hígado y 4 en mesenterio. Determinando así que el parásito C. tenui- collis afecta en mayor cantidad al epiplón en todas las razas seguidas del mesenterio y peritoneo con menor frecuencia y al hígado una pequeño grupo de animales.

Tabla 4 Órganos o estructuras anatómicas afectadas, de acuerdo a la raza

\begin{tabular}{lcccccc}
\hline Razas & \# Epiplón & \# Hígado & \# Mesenterio & \# Peritoneo & $\mathbf{X}^{\mathbf{2}}$ & P-valor \\
\hline Criolla & 52 & 3 & 14 & 13 & & \\
Merino & 29 & 4 & 8 & 2 & & \\
Mestiza & 17 & - & 5 & - & 6.79 & 0.6587 \\
Corriedale & 11 & 1 & 4 & & \\
\hline
\end{tabular}

Tabla 5 Órganos y estructuras anatómicas afectadas de acuerdo al sexo

\begin{tabular}{lcccccc}
\hline Razas & \# Epiplón & \# Hígado & \# Mesenterio & \# Peritoneo & $\mathbf{X}^{\mathbf{2}}$ & P-valor \\
\hline Macho & 46 & 6 & 12 & 11 & & \\
Hembra & 63 & 2 & 19 & 11 & 3.93 & 0.2687 \\
\hline
\end{tabular}

Tabla 6 Prueba de Chi 2 de significancia para la asociación o independencia entre la frecuencia de casos y la raza

\begin{tabular}{lcccccc}
\hline Raza & $\mathbf{N}^{\circ}$ Examinados & $\mathbf{N}^{\circ}$ Infestados & Prevalencia (\%) & IC 95\% & $\mathbf{X}^{2}$ & P-valor \\
\hline Criolla & 168 & 65 & 38.69 & $31.6-46.4$ & & \\
Merino & 109 & 35 & 32.11 & $23.2-40.8$ & & \\
Mestiza & 51 & 21 & 41.18 & $27.5-54.4$ & & \multirow{2}{*}{ Corriedale } \\
\hline
\end{tabular}

Tabla 7 Prueba de Chi2 de significancia para la asociación o independencia entre la frecuencia de casos y la procedencia

\begin{tabular}{|c|c|c|c|c|c|c|}
\hline Procedencia & $\mathbf{N}^{\circ}$ Examinados & $\mathrm{N}^{\circ}$ Infestados & Prevalencia (\%) & IC 95\% & $\overline{X^{2}}$ & P-valor \\
\hline Izamba & 104 & 37 & 35.58 & $26.8-42.2$ & & \\
\hline Guaranda & 30 & 11 & 36.67 & $19.7-54.3$ & & \\
\hline Pelileo & 25 & 10 & 40.00 & $20.8-59.2$ & & \\
\hline Pilahuin & 23 & 8 & 34.78 & $33.0-36.9$ & & \\
\hline Quisapincha & 93 & 39 & 41.94 & $31.9-52.0$ & & \\
\hline SM. Salcedo & 67 & 24 & 35.82 & $24.5-47.5$ & & \\
\hline Santa Rosa & 20 & 4 & 20.00 & $2.5-37.5$ & 3.73 & 0.713 \\
\hline
\end{tabular}


Figura 1 Zona de aceptación de Ho de acuerdo a la raza

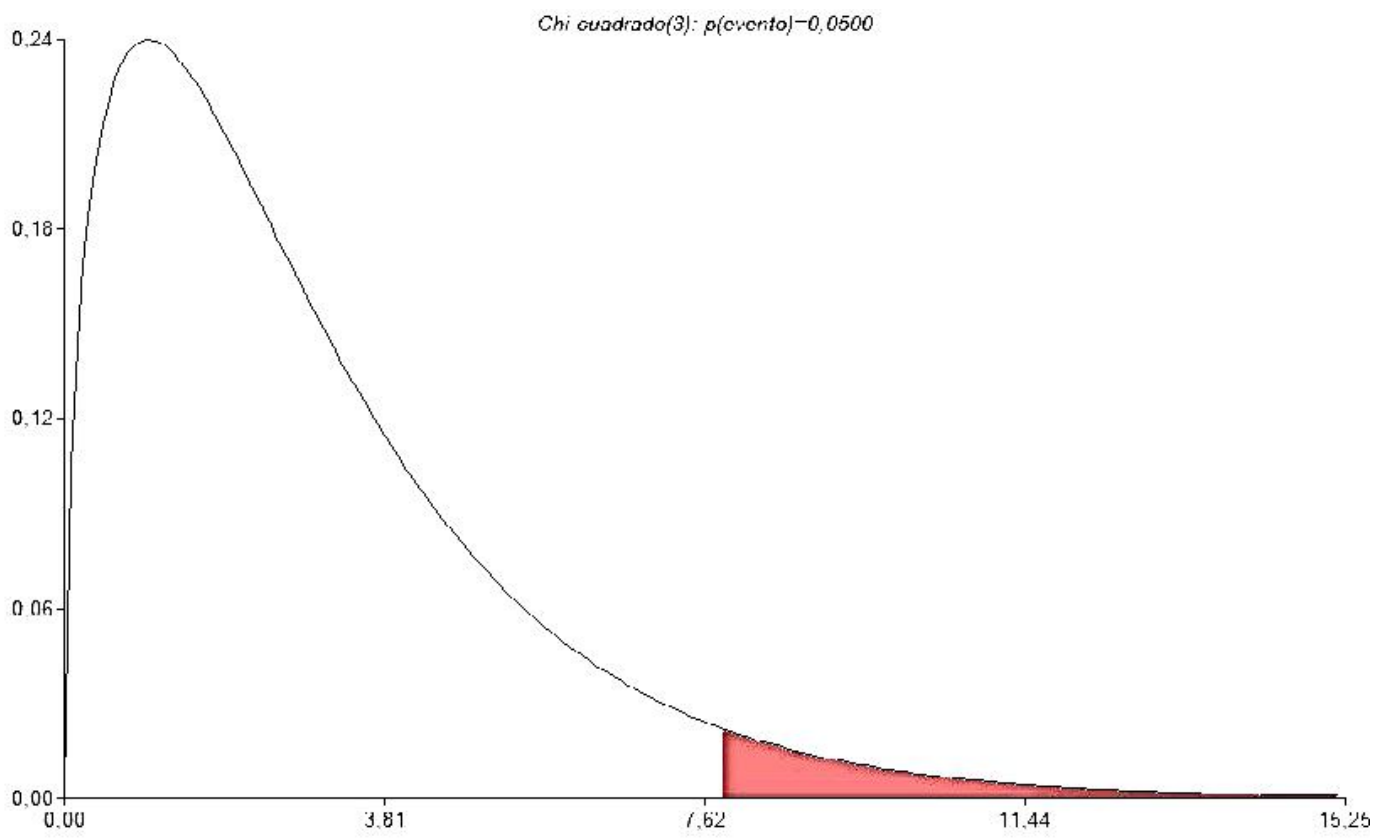

Figura 2 Zona de aceptación Ho de acuerdo al sexo

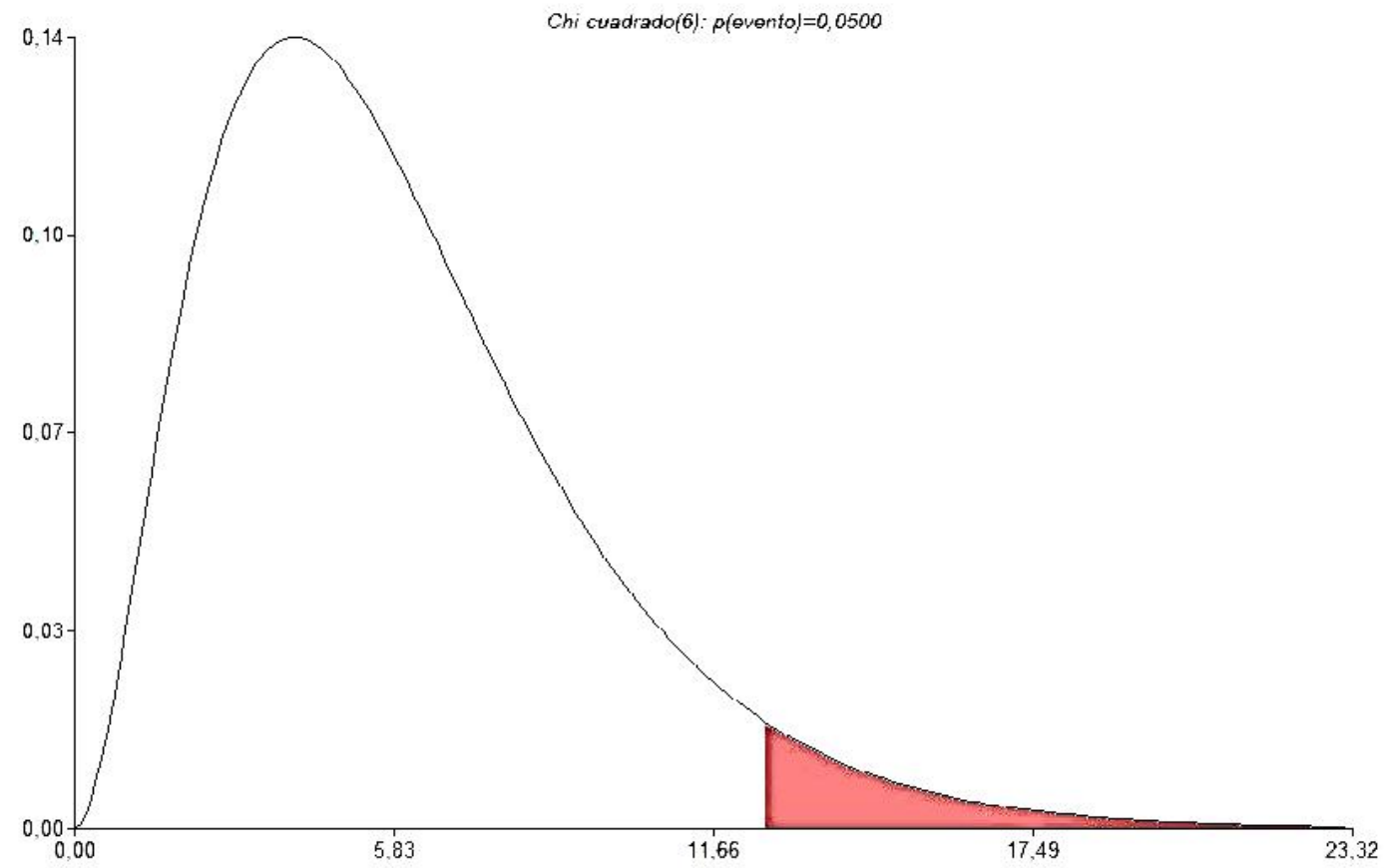




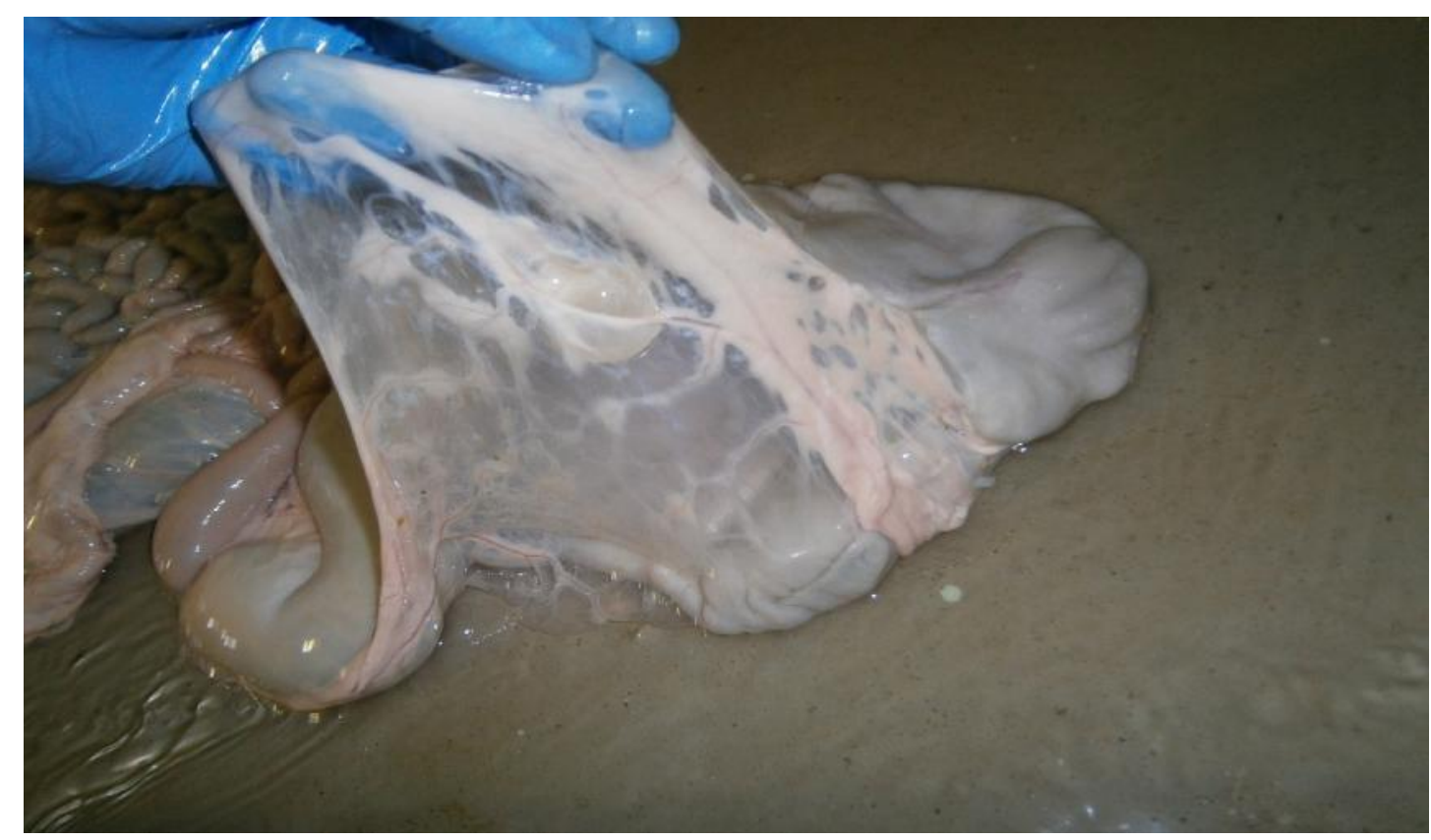

Figura 4 Cysticercus tenuicollis de diferentes tamaños

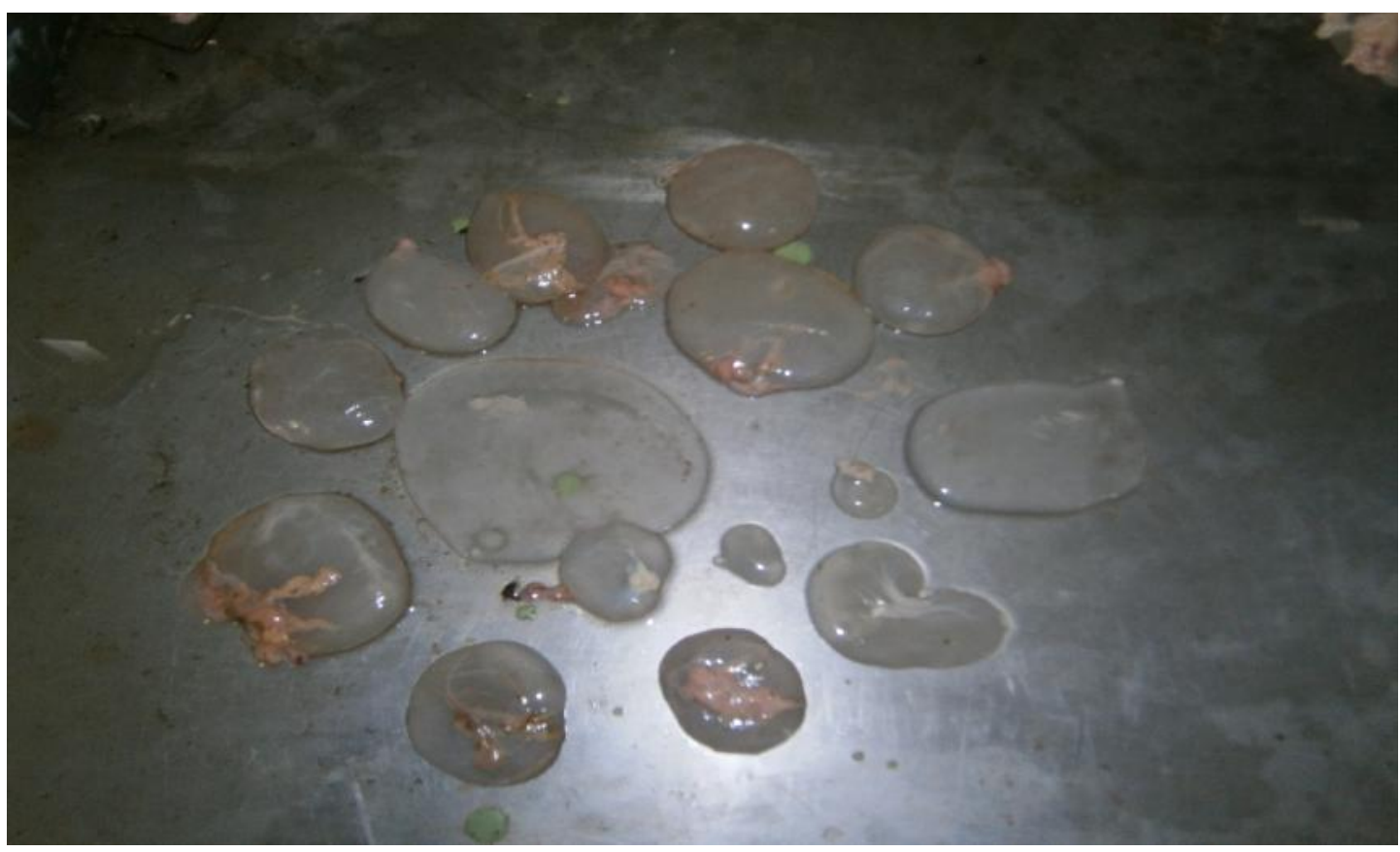

La tabla 5 indica que en el epiplón fue la estructura más afectada por la presencia del C. tenuicollis, 46 machos y 63 hembras. La segunda estructura más afectada fue el mesenterio con 12 machos y 19 hembras infestadas, el peritoneo fue afectado con 11 animales macho y hembra respectivamente por el 
parásito, en el hígado hubo 6 machos y 2 hembras infestadas. Determinando que el epiplón es la estructura predilecta del parásito tanto en hembras como en machos.

Mediante la prueba estadística (Chi cuadrado), Tabla 6 , se determinó que el valor de $\mathrm{X}^{2}$ calculado es de 1.74 , valor que es menor al de $\mathrm{X}^{2}$ tabulado que es de 7.82 Figura 1, con 3 GL. Por consiguiente se determina que la raza de los ovinos faenados no influye en la prevalencia de $C$. tenuicollis en su cavidad abdominal. Mediante la prueba (Chi cuadrado), Tabla 7 , se determinó que el valor de $X^{2}$ calculado es de 3.73 , valor que es menor al de $\mathrm{X}^{2}$ tabulado que es de 12.59 Figura 2, con 6 GL. Por consiguiente se determina que la procedencia de los ovinos faenados no influye en la prevalencia de $C$. tenuicollis en su cavidad abdominal. Cysticercus tenuicollis presente en epiplón Figura 3 y Cysticercus tenuicollis de diferentes tamaños Figura 4.

\section{Discusión}

Una vez concluida la investigación para determinar la prevalencia de $C$. tenuicollis en cavidad abdominal de ovinos faenados en el camal municipal del cantón Ambato, los resultados señalan lo siguiente: En la población de ovinos sacrificados en el camal se observó que el órgano más afectado fue el epiplón en los de animales infestados con un $64 \%$ por tal motivo concuerdo con (Saulawa et al. 2011) en cuyo estudio señala que los sitios de predilección del C. tenuicollis en varios órganos viscerales como epiplón con una prevalencia (35.29\%) seguido de los pulmones. Al mismo tiempo nuestros datos no concuerdan con el mismo autor puesto que la prevalencia de $C$. tenuicollis es mayor en los ovinos de sexo macho, en el estudio realizado en el camal de Ambato no hubo significancia y se determinó que el 113 parásito afecta a ambos géneros por igual en el epiplón principalmente. Por otro lado (Radfar et al. 2005) determinó que animales sacrificados se observó que el sitio predominante de los cisticercos fue el epiplón, en el ganado ovino con el $84 \%$ y cabras con un $82 \%$. Se determinó que los cisticercos de ovino y caprino, probablemente representan dos cepas diferentes y posiblemente siga el mismo patrón de especiación como se informa en el Echinococcus granulosus. Al igual (Radfar et al. 2005) informa en su estudio que en los animales que fueron sacrificados se observó que el sitio predominante y de predilección de cisticercos en el ganado ovino con un $84.85 \%$ y cabras con $82.14 \%$ con una diferencia significativa de 0.05 fue el epiplón la estructura anatómica más afectada, nuestros datos concuerdan con este autor. Por otro lado la investigación que realizó (Mekuria et al. 2013) también demuestra que el quiste se localizó con mayor frecuencia conectado al hígado, epiplón y el peritoneo en ovino y caprino. Determinando que, la presencia de $C$. tenuicollis con una prevalencia más alta y el consiguiente efecto sobre los pequeños rumiantes significan que existe la necesidad de controlar la población de perros callejeros, llevando un registro de desparasitación, además se debe evitar el faenamiento de tras patio y clandestino, la eliminación de las vísceras infectadas para reducir el problema.

Mediante el cálculo de frecuencias absolutas y porcentuales se determinó que en base a la proporción de animales infestados la raza Mestiza con un 41.18 $\%$, son los animales con mayor porcentaje de $C$. tenuicollis identificados mediante la inspección post mortem de los ovinos faenados. Seguidos de la raza Criolla con un $38.69 \%$, Corriedale con $35.29 \%$ y Merino con $32.11 \%$. Coincidiendó con el estudio que realizó (Garijo-Toledo et al. 2005), titulado Cestodosis larvarias en el ganado ovino de la región 
de Murcia (sureste de España), se lo realizo con la intención de conocer el estado parasitario del ganado ovino adulto, en relación a las cestodosis larvarias. Para tal fin, se examinaron un total de 151 ovejas adultas sacrificadas en un matadero regional, 18 animales $(11.92 \%)$ presentaron la presencia de $C$. tenuicollis, en los animales sacrificados se observó que las vesículas estaban localizadas en membranas serosas de la cavidad abdominal y superficie hepática. La identificación y presencia de cisticercos coincidió con el hallazgo de lesiones hepáticas a modo de trayectos fibróticos sinuosos en mayor o menor grado.

De acuerdo con los datos obtenidos se demuestra que la raza y el sexo no son dependientes para la presencia de $C$. tenuicollis en su cavidad abdominal y consecuentemente no interfiere en la aparición del parásito en algún órgano o estructura anatómica en específico. Indicando que el parásito puede desarrollarse por igual en todos los órganos del individuo sin depender de la sexo y raza. En el estudio Realizado por (Saulawa et al. 2011), se realizó el estudio prospectivo, mediante la inspección post mortem de ovejas sacrificadas en el matadero, para detectar la presencia de quistes (C. tenuicollis), los resultados de una población total de 261 ovejas examinados, 34 ovinos fueron positivos $13 \%$ de animales infectados, se determinó la prevalencia de la infección aumenta con la edad, 24 ovinos tenían más de 24 meses representaron el 39\% de la población. Al igual que el sexo, los machos tenían relativamente mayor prevalencia que las hembras. Los datos registrados señalan que los sitios de predilección del $C$. tenuicollis en órganos viscerales, el epiplón que tiene la prevalencia (35.29\%), pulmones (11.76\%). Los resultados sugieren que $C$. tenuicollis es común $\mathrm{y}$ puede constituir un problema de salud en el ganado ovino y una fuente de pérdidas económicas en la industria de la carne.

\section{Conflictos de intereses}

Los autores declaran que no existen conflictos de interés.

\section{Agradecimientos}

Nuestro reconocimiento profundo a la Universidad Técnica de Ambato Carrera de Medicina Veterinaria y Zootecnia, y a los camales frigoríficos de la provincia de Tungurahua por permitirnos realizar la investigación en sus instalaciones.

\section{Literatura citada}

Atto-Mendives JA. 2007. Importancia de los ovinos tropicales introducidos al país: características productivas y reproductivas. Arch Latinoam Prod Anim. 2007; (15) (Suppl 1):310-5.

Borja-Caicedo BE. Prevalencia de la hidatidosis en cerdos faenados en el camal Municipal de la ciudad de Ambato. [Tesis de Licenciatura]. Universidad Técnica de Ambato. Ecuador. 2013. p. 66.

Frontera-Carrión EM, Bravo D, Blanco J, Herrador P, Calero R, Serrano F, et al. Las Parasitosis Porcinas y sus Repercusiones Económicas. Suis 2012; 87: 18-27.

Garijo-Toledo MM, Alonso FD, Martínez-Carrasco C, Ruiz de Ibáñez MR. Cestodosis larvarias en el ganado ovino de la región de Murcia (sureste de España). Murcia, España. Res Rev Parasitol 2005; 65(1-4): 43-7.

González H. 1982. Pérdidas económicas producidas por las parasitosis de los rumiantes. VIII Jornadas Médico Veterinarias. Universidad Austral de Chile. Valdivia. 1982. p. 39-48. 
Kahl-Martin C. Fundamentos de epidemiología. Edi ciones Díaz de Santos. 1ed. 1990. Medellin. Colombia. 1990. p. 368.

Mekuria E, Shimelis Sh, Bekele J, Sheferaw D. Sheep and goats Cysticercus tenuicollis prevalence and associated risk factors. Afr J Agric Res 2013; 8(24): 3121-5.

Quiroz-Romero H, Figueroa-Castillo JA, IbarraVelarde F, López-Arellano ME. Epidemiologia de enfermedades parasitarias en animales domésticos. 1ed. México, DF. 2011. p. 643.

Quiroz-Romero H. Parasitología y enfermedades parasitarias en animales domésticos. México, DF. Limusa. 1984. p. 870.
Radfar MH, Tajalli HS, Jalalzadeh M. Prevalence and morphological characterization of Cysticercus tenuicollis (Taenia hydatigena cysticerci) from sheep and goats in Iran. Vet Arh. 2005; 75(6): 469-76.

Saulawa MA, Magaji AA, Faleke OO, Mohammed AA, Kudi AC, Musawa AL, et al. Prevalence of Cysticercus tenuicollis cysts in sheep slaughtered at Sokoto abattoir, Sokoto state, Nigeria. Sokoto J Vet Sci. 2011; 9(2):24-7.

Vega-Pérez CA, Garcia-Barrera DR. Guía práctica para pequeños productores ovinos. Fundación social de Holcim Colombia. 2011. Consultado 03 de Junio. Disponible en:http://www. fundacionsocialholcimcolombia.org/OVINOS_GuiaPractica.pdf. 